\title{
Design and analysis of computer experiments for efficient model-based active thermography in the agro-food sector
}

\author{
by K. D’huys ${ }^{*}{ }^{* *}$, B. De Ketelaere* ${ }^{*}$ J. C. Keresztes* and W. Saeys* \\ * Division MeBioS, Department Biosystems, KU Leuven, Kasteelpark Arenberg 30, B-3001 Heverlee, Belgium \\ ** Corresponding author : Karlien.Dhuys@biw.kuleuven.be
}

\begin{abstract}
Finite element models simulating the heat transfer in active thermography are studied intensively. Together with improved model accuracy, the related complexity and required computational power are increasing. In the agro-food sector, the material properties of the inspected objects, and thus the input parameters of heat transfer models, are prone to biological variability. In this work, a strategy called 'Design and Analysis of Computer Experiments' was applied to efficiently obtain the heat transfer output for an arbitrary combination of input parameters without running every single simulation. A metamodel was set up, interpolating between the output of a few carefully selected simulations.
\end{abstract}

\section{Introduction}

In active infrared thermography (AT), a thermal excitation is applied to the inspected object and an infrared camera is used to monitor how the surface temperature of the object responds to this excitation as a function of time. By creating a transient heat flow, (sub)surface defects associated with deviations in thermo-physical properties will result in surface temperature contrasts which can be detected in a non-destructive and contactless way [1,2]. Depending on the waveform that is used by the heat source to deliver the excitation energy, several types of AT can be distinguished. The two main types are pulsed thermography, in which a heat pulse is applied to the sample surface, and lock-in thermography, in which sinusoidal thermal waves are emitted by the excitation source [3]. AT is being studied intensively in several domains, such as building diagnostics [4], inspection of automotive and airplane parts [5], medical screening [6], inspection of artwork and historical monuments [7], etc. Several defect types can be detected, such as delaminations, cracks, voids and foreign material inclusions [3]. In addition to defect detection, active thermography also allows for defect quantification (e.g. defect depth, defect diameter) [8]. While most initial studies on AT adopted an empirical approach, in the last decade, focus has shifted towards modeling and fully understanding the underlying heat transfer processes [9-11]. There are several motivations to model the heat transfer processes occurring during an AT experiment, including to verify experimental observations, to optimize the experimental settings to inspect a certain sample, to determine a sample's thermo-physical properties, etc [11]. In general, heat transfer simulations provide a way to reduce the workload and costs associated with physical experimentation. AT models described in literature vary in terms of their dimension (1D, 2D, 3D), the complexity of the studied geometry and the mechanisms of heat transfer included in the model. Moreover, several numerical methods have been described to solve the heat transfer problems, e.g. the Finite Element method, the Finite Volume method, etc [10]. Although in many cases computer simulations provide an efficient alternative to physical experiments, the computation time required to perform such simulations might become considerably high when complex geometries or heat transfer mechanisms (e.g. radiation) are considered.

In the agro-food sector, the demand for online quality inspection tools is increasing rapidly. In order to be eligible for online application, such tools should be contactless, non-destructive and fast. For some applications no suitable technique was developed thus far. Because the defects that can be detected using AT in the higher mentioned domains are similar to certain important quality characteristics to be monitored in the agro-food sector, the method could also be very valuable for this sector. However, few reports were found on AT applications in the agro-food domain [1214]. Since false negatives should be reduced to the absolute minimum in food safety and quality inspection, a full understanding of the applied AT methodology and the obtained results is crucial. Computer simulations of the involved heat transfer processes may provide this understanding. Since food products are complex and prone to biological variability, however, models will need to be of sufficient complexity to fully grasp the phenomena occurring during their inspection. As stated earlier, increased model complexity entails increased computation times.

In order to approach these simulations or computer experiments in an efficient way, the concept of 'Design and Analysis of Computer Experiments' (DACE) was proposed in 1989 by Sacks et al. [15]. The DACE approach comprises four main steps. In a first step, the variables that make up the so-called 'design space' have to be identified and for each of these variables, the range of possible numerical values should be defined. In a second step, the combinations of input parameters at which simulations should be performed, the 'design points', have to be selected. This selection is typically done according to space filling designs that sample the design space evenly, e.g. Latin hypercube designs [16]. In a third step, simulations have to be performed at the design points. In a fourth and final step, a Gaussian Process (GP) interpolation between the simulation outputs is used to create the metamodel [17]. This interpolation model can then be used to obtain the simulation output at any arbitrary combination of input parameters within the design space, without actually having to run the computationally intensive simulation. The DACE approach is based on the fact that most 
computer experiments have a deterministic output, eliminating the need for replication in the design. By avoiding replication, a more efficient screening of the design space is obtained with the same number of simulations performed [15].

This study aims at providing an efficient method for heat transfer modeling in AT, based on the DACE framework. The approach is combined with a Principal Component Analysis (PCA) of thermal response profiles. PCA allows to project the thermal response onto a very limited number of underlying factors. The method was developed and validated for a PVC sample with measured thermo-physical properties. In this way, a strong basis for model-based AT in agro-food applications was provided.

\section{Experimental set-up}

\subsection{Sample}

The DACE strategy was developed for a finite element model of the heat transfer processes occurring in a polyvinyl chloride (PVC) sample with flat bottom hole defects. Since 2D models were set up, the defects were created in the form of grooves along the entire width of the sample, instead of as circular defects. In this way, the similarity between the real and modeled geometry was maximized. Defects with a subsurface depth of $1 \mathrm{~mm}, 2 \mathrm{~mm}$ and $3 \mathrm{~mm}$ were considered. Details on the sample geometry are provided in figure 1. The thermo-physical properties of the PVC material were measured and are displayed in table 1 . The thermal conductivity and heat capacity were determined using the guarded hot plate method (ASTM standard C177) and a differential adiabatic scanning calorimeter (dASC) (patent no. WO/2012/103601), respectively $[18,19]$. The surface emissivity was evaluated with the noncontact thermometer method as described in ASTM standard E1933-14 [20].

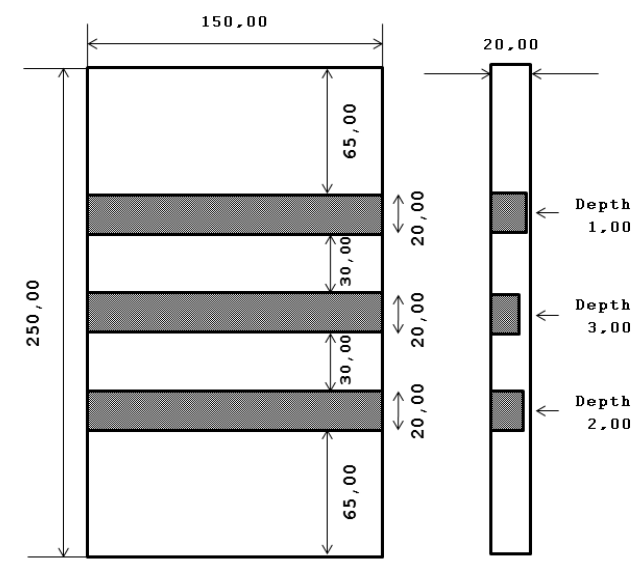

Table 1. Thermo-physical properties of PVC

\begin{tabular}{|c|c|}
\hline Property & Value \\
\hline $\begin{array}{l}\text { Thermal conductivity } k \\
{[\mathrm{~W} /(\mathrm{m} \cdot \mathrm{K})]}\end{array}$ & $0.148\left(\right.$ at $\left.\mathrm{T}=20^{\circ} \mathrm{C}\right)$ \\
\hline Density $\rho\left[\mathrm{kg} / \mathrm{m}^{3}\right]$ & 1415 \\
\hline $\begin{array}{c}\text { Heat capacity } C_{p} \\
{[\mathrm{~J} /(\mathrm{kg} \cdot \mathrm{K})]}\end{array}$ & $842\left(\right.$ at $\left.\mathrm{T}=20^{\circ} \mathrm{C}\right)$ \\
\hline Surface emissivity & 0.94 \\
\hline
\end{tabular}

Fig. 1. PVC sample geometry (all dimensions are in $\mathrm{mm}$ )

\subsection{Pulsed thermography set-up}

The PVC sample described in section 2.1 was placed in a thermally inert sample holder and inspected in reflection mode using a cooled FLIR SC7600 with an InSb detector, a spatial resolution of 640x512 pixels and a noise equivalent temperature difference (NETD) of $<25 \mathrm{mK}$ (FLIR Systems Inc., USA). Two halogen lamps with a maximum power of $2500 \mathrm{~W}$ (Hedler $\mathrm{GmbH}$, Germany), controlled by a signal generator (edevis $\mathrm{GmbH}$, Germany), were used as excitation sources. The lamps were positioned in such a way that maximum uniformity of the excitation was obtained. To further improve uniformity, the lamps were equipped with frosted glass diffusers and metal reflectors (Hedler $\mathrm{GmbH}$, Germany). The thermal camera was equipped with a long-pass IR filter (Spectrogon Inc., USA) to avoid reflections in the thermal images by separating the excitation wavelength range from the wavelength range of the IR sensor. A pulsed thermography experiment was performed in which a $5 \mathrm{~s}$ heat pulse was applied and frames were recorded for $120 \mathrm{~s}$ at a frame rate of $10 \mathrm{~Hz}$. The software package DisplaylMG (edevis $\mathrm{GmbH}$, Germany) was used for synchronization of the halogen lamps and the camera and for recording.

\section{Heat transfer modeling}

Three 2D finite element models of the heat transfer occurring during the pulsed thermography experiment described in section 2.2 were set up in Comsol Multiphysics ${ }^{\circledR}$ (Comsol Inc., USA). The models differ in their level of complexity and consequently in the computation time required to solve them. In a first model, the pulsed excitation was considered as a boundary heat flux, and surface-to-ambient radiative cooling and convective cooling boundary conditions were set. Convection coefficients were calculated from correlation formulas for external natural convection based on the Rayleigh number [21]. In a second model, surface-to-ambient radiation and convection boundary conditions were identical to the ones in the first model, yet the excitation was modeled as a prescribed radiosity originating from the front 


\subsection{1/qirt.2016.026}

surface of a heat source. Surface-to-surface radiation between PVC sample and heat source was also considered. However, since these two models did not provide an accurate approximation of the cooling profile of both sound and defective regions of the PVC sample, they are not described in detail. A third, more complex model, is described below and was used in further analysis.

\subsection{Geometry and material properties}

Figure 2 illustrates the geometry considered in the most complex model, in which a box of air surrounds the PVC sample and a halogen lamp to be able to study convective heat transfer in the area surrounding the sample. The material properties shown in table 1 were assigned to the PVC sample, the temperature- and pressure-dependent material properties of air (extracted from the Comsol material library) were assigned to the box of air.

\subsection{Physics}

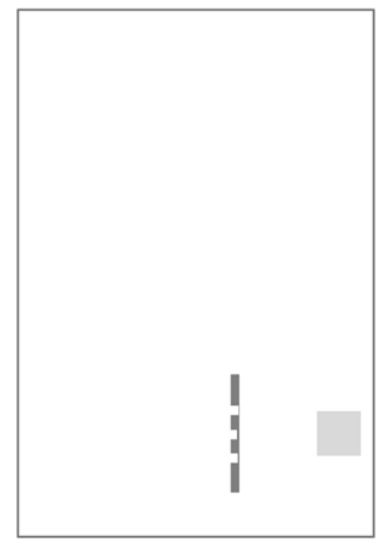

Fig. 2. Geometry of most complex model

\subsubsection{Heat transfer}

The governing equation to be solved on the model domain is the conductive heat transfer equation, defined as:

$$
\rho C_{p} \frac{\partial T}{\partial t}+\nabla \cdot(-k \nabla T)=0
$$

with $T$ the absolute temperature $[K], \rho$ the density $\left[\mathrm{kg} / \mathrm{m}^{3}\right], C_{p}$ the heat capacity at constant pressure $[\mathrm{J} /(\mathrm{kg} \cdot \mathrm{K})], \mathrm{k}$ the thermal conductivity $[\mathrm{W} /(\mathrm{m} \cdot \mathrm{K})]$ and $t$ the time $[\mathrm{s}]$ [22]. The initial temperature, $T_{0}$, was set at $17.28^{\circ} \mathrm{C}$, as obtained experimentally. A surface-to-ambient radiation boundary condition (Eq. (2), with $\varepsilon$ the surface emissivity, $\sigma$ the StefanBoltzmann constant and $T_{a m b}$ the ambient temperature) was applied to all boundaries that were not within the view factor of other boundaries. A surface-to-surface radiation boundary condition (Eq. (3), with $\varepsilon$ the surface emissivity, $G$ the irradiation $\left[\mathrm{W} / \mathrm{m}^{2}\right]$ and $\sigma$ the Stefan-Boltzmann constant) was applied to all boundaries that were within the view factor of other boundaries. A prescribed radiosity $J_{0}\left[\mathrm{~W} / \mathrm{m}^{2}\right]$ in the positive normal direction was applied to the front surface of the halogen lamp. Since its amplitude was unknown, it was selected to obtain the same temperature at $t=20 \mathrm{~s}$ at the sound region of the sample surface in simulation and experiment. This way, a prescribed radiosity of $8175 \mathrm{~W} / \mathrm{m}^{2}$ was obtained.

$$
\begin{aligned}
& -n \cdot(-k \nabla T)=\varepsilon \sigma\left(T_{a m b}^{4}-T^{4}\right) \\
& -n \cdot(-k \nabla T)=\varepsilon\left(G-\sigma T^{4}\right)
\end{aligned}
$$

\subsubsection{Non-isothermal flow}

In order to model natural convection in the box of air surrounding the sample, the Navier-Stokes equation for a compressible, Newtonian fluid (Eq. (4)) was solved together with the continuity equation (Eq. (5)):

$$
\begin{aligned}
& \rho \frac{\partial u}{\partial t}+\rho(u \cdot \nabla) u=\nabla \cdot\left[-p I+\mu\left(\nabla u+(\nabla u)^{T}\right)-\frac{2}{3} \mu(\nabla \cdot u) I\right]+F \\
& \frac{\partial \rho}{\partial t}+\nabla \cdot(\rho u)=0
\end{aligned}
$$

with $u$ the fluid velocity $[\mathrm{m} / \mathrm{s}], p$ the fluid pressure $[\mathrm{Pa}], \rho$ the fluid density $\left[\mathrm{kg} / \mathrm{m}^{3}\right], \mu$ the fluid dynamic viscosity $[\mathrm{Pa} \cdot \mathrm{s}]$ and $F$ the external forces applied to the fluid [23]. 
Equation (6) governs the heat transfer in the air box:

$$
\rho C_{p} \frac{\partial T}{\partial t}+\nabla \cdot(-k \nabla T)+\rho C_{p} u \cdot \nabla T=Q
$$

with $\rho, C_{p}$ and $k$ the density $\left[\mathrm{kg} / \mathrm{m}^{3}\right]$, heat capacity at constant pressure $[\mathrm{J} /(\mathrm{kg} \cdot \mathrm{K})]$ and thermal conductivity $[\mathrm{W} /(\mathrm{m}$. $K)]$ of the fluid, $T$ the absolute temperature $[K]$ and $u$ the fluid velocity $[\mathrm{m} / \mathrm{s}]$.

The initial fluid velocity was set to be zero in both $x$ - and y-direction and the air initially was at atmospheric pressure. A no-slip boundary condition was applied to the PVC sample and halogen lamp boundaries. The outer boundaries of the air box were assigned as being open boundaries and a gravity term in the y-direction was applied to the air-domain.

\subsection{Mesh and study steps}

The PVC sample was meshed using a mapped mesh, with a resolution of 4 elements per mm of thickness ( $x$ direction). Halogen lamp and air box were meshed using a triangular mesh. The mesh had a total number of elements of 106 357. The study was solved in two time-dependent steps. In a first step (0-5 s), a prescribed radiosity was originating from the halogen lamp surface. In the second step (5.1-120 s), this prescribed radiosity was enabled. A time-step of $0.1 \mathrm{~s}$ was used in both steps. The computation time for a single simulation was $1828 \mathrm{~s}$ using a $3.40 \mathrm{GHz}$ processor.

\section{Design and Analysis of Computer Experiments}

The Latin hypercube design, Principal Component Analysis and Gaussian process modeling were performed in JMP Pro 12 (SAS Institute Inc., USA). Heat transfer simulations were performed in Comsol Multiphysics ${ }^{\circledR}$ through the LiveLink $^{\text {TM }}$ for Matlab® (The Mathworks Inc., USA). The comparison of temperature-time profiles was performed in Matlab®.

\subsection{Design space}

The material properties of PVC were used as input parameters in the interpolation model. Since $C p$ and $\rho$ always occur as a product in the heat transfer equation, the volumetric heat capacity $\rho C p$ was considered as a single variable. The limits of the design space are shown in table 2. For each material property, a range of values was considered that contains the reference values.

\subsection{Space filling design}

A maximin space filling Latin hypercube design of 30 runs (=unique combinations of the input parameters) was created and is illustrated in figure 3. This type of design maximizes the minimum distance between points in the design space, while maintaining an even spacing between factor levels [16]. The number of runs was selected based on the rule of thumb to use 10 times the number of input parameters. Since the results of computer experiments are deterministic, no replications were included in the design [17].

Table 2. Reference value and limits of the design space for each of the input parameters

\begin{tabular}{ccc}
\hline Property & Reference & Range of values \\
\hline $\begin{array}{c}\text { Thermal conductivity } \\
k[W /(m \cdot K)]\end{array}$ & 0.148 & $0.1-0.2$ \\
$\begin{array}{c}\text { Volumetric heat } \\
\text { capacity }\end{array}$ & 1191430 & $834002-1548859$ \\
$\rho C_{p}\left[J /\left(m^{3} \cdot K\right)\right]$ & \\
Surface emissivity & 0.94 & $0.7-1$ \\
\hline
\end{tabular}

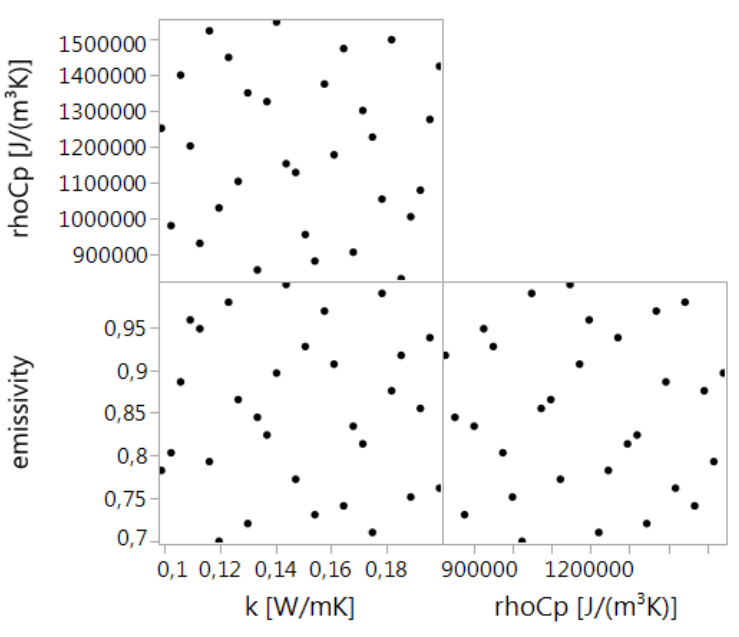

Fig. 3. Latin hypercube design of 30 runs 


\subsection{1/qirt.2016.026}

\subsection{Defining the model response}

At each of the combinations of input parameters defined by the design points in the space filling design, a heat transfer simulation was performed and the temperature-time profile for the sound region was extracted. Based on the thus obtained dataset of 30 temperature-time profiles, a Principal Component Analysis (PCA) was performed.

PCA is a multivariate data analysis technique that allows to reduce a data set's dimensionality. It transforms a large set of correlated variables $X$ to a smaller set of uncorrelated principal components (PC's) while still describing most of the variation in the data. The model structure for PCA is given by (Eq. 7):

$$
X=T P^{\prime}+E
$$

with $X$ the mean-centered original data set, $T$ the scores matrix, $P^{\prime}$ the transposed loadings matrix and $E$ the noise or undescribed variability in the data. The scores matrix $T$ contains the A most dominant PC's of $X$. The PC's are independent linear combinations of all elements in $X$. They correspond to the directions of the data in which the variance is largest and PC's are orthogonal to each other. The PC's are ordered with respect to the amount of variation in the original variables they explain. The number of components $A$ is selected based on the desired amount of variation in the data to describe. The loadings matrix $P$ ' provides information on how the variables in $T$ relate to the original data in $X$ [24, 25].

In this study, the scores of the principal components describing the larger part of the variability in the temperature-time profiles were used as responses in the DACE model. A Gaussian process model was built for each of the responses, i.e. for each of the principal component scores included in the PCA model.

\subsection{Gaussian process modeling and validation}

After performing heat transfer simulations at the design points defined in section 4.2 and obtaining the values of the responses (section 4.3) at each of these design points, a Gaussian process (GP) model was set up for each response. This model interpolates between the values of that response at the design points and predicts this value at every possible combination of input parameters within the limits of the design space.

GP models interpolate the provided data perfectly, i.e. no error is assumed at the design points. The deterministic responses are considered as the realization of a random stochastic, multivariate normal process and are represented as $y(x)$, an $n \times 1$ data vector with $n \times 1$ mean $\mu 1_{n}$ and a covariance matrix defined as:

$$
\operatorname{Var}(y)=\sigma^{2} R(X, \theta)
$$

in which a Gaussian $n \times n$ correlation matrix $R(X, \theta)$ is used and is expressed as follows:

$$
R_{i j}(X, \theta)=\exp \left(-\sum_{k} \theta_{k}\left(x_{i k}-x_{j k}\right)^{2}\right)
$$

wherein $\theta_{k} \geq 0$ describes the correlation across the range of factor $k$ and gives information on the flatness or bumpiness of the fitted surface in the $k^{\text {th }}$ direction. A maximum likelihood estimation of $\mu, \sigma$ and $\theta$ is performed and predicted values for the responses $y(x)$ are calculated within the design space [17]. A more detailed description of the GP interpolation model can be found in [17].

Since an inherent characteristic of GP models is that the prediction error increases with increasing distance from the design points, we can state that when the model predicts the principal component scores well at points that are at maximum distance from the design points, the model performance is acceptable along the entire design space. According to this reasoning, a space filling augmentation of the original LHS design with 10 extra design points was performed and the GP model was validated at these additional points. The design augmentation was done using the fast flexible filling method with the maximum projection (MaxPro) criterion. The MaxPro criterion maximizes the product of the distances between design points while involving all factors [26]. The temperature-time profiles of the sound region were determined performing FEM simulations at the augmented design points and compared to the temperature-time profiles reconstructed based on the PC1 and PC2 scores predicted by the GP model for these points. The goodness of fit of FEM simulated and reconstructed profiles was evaluated by calculating the sum of squared error (SSE) between them. These SSE-values were compared relative to the SSE calculated between each FEM simulated profile and the mean FEM simulated profile to give a better appreciation of the model accuracy. 


\section{Results and discussion}

\subsection{Reference versus simulated temperature-time profiles}

Reference profiles of temperature versus time for sound and defective regions were obtained by averaging profiles of three repeated measurements and of a selection of pixels for each region (168 pixels for each of the defects and 6924 pixels for the sound region). The resulting temperature-time profiles are shown in figure 4 (left). Temperaturetime profiles obtained from finite element simulations are shown in figure 4 (right). Although there is a clear overshoot in the maximum temperature for the simulated profiles, the thermal decay shows a similar behaviour in the experiments and the simulations. The overshoot is presumably to be attributed to the dynamics of the thermal camera.
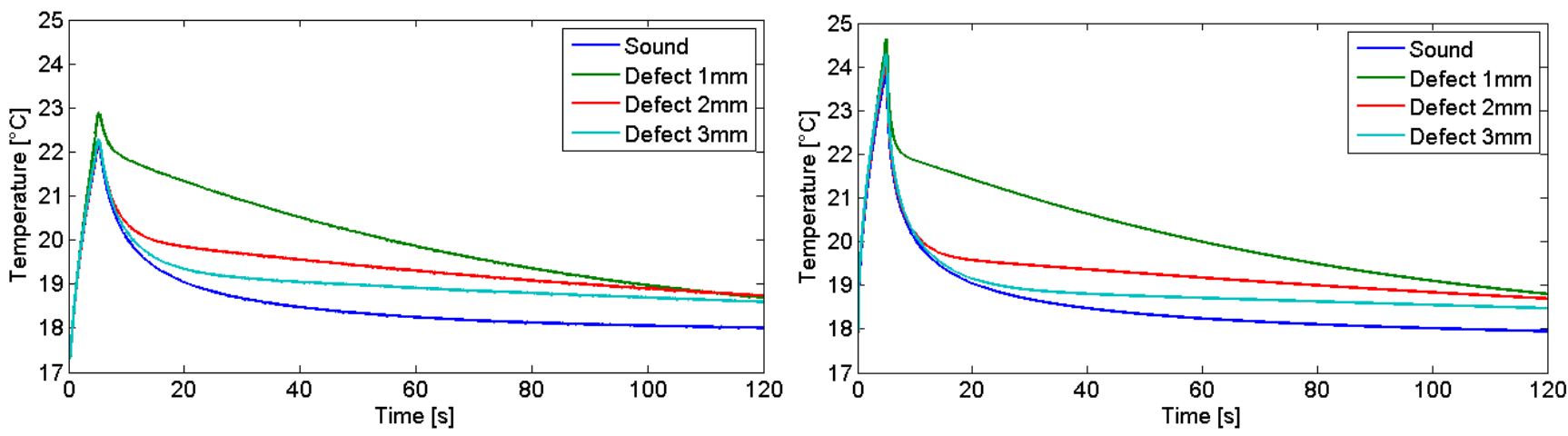

Fig. 4. Experimentally obtained (left) and simulated (right) temperature-time profiles for sound and defective regions

\subsection{Design and Analysis of Computer Experiments}

\subsubsection{Principal Component Analysis}

Principal Component Analysis was performed on the 30 mean-centered temperature-time profiles obtained by performing FEM simulations at the design points. In figure 5 (left) a biplot of PC1 versus PC2 is shown for this PCA model. The variation in the scores (black dots) is largest along the direction of PC1. The positioning of the loadings (red arrows) indicates that the variables (temperature values at 1200 time steps within the temperature-time profiles) are highly correlated. Figure 5 (right) shows the loadings of PC1 and PC2 as a function of time. The loadings plot for the first $\mathrm{PC}$ has a similar shape as the temperature-time profiles and provides information on the amplitude of the profiles. Only the two first principal components (PC1 and PC2) were retained in the model, since together they explain $99.994 \%$ of the variance in the dataset. These two principal components were used as responses in the DACE analysis.
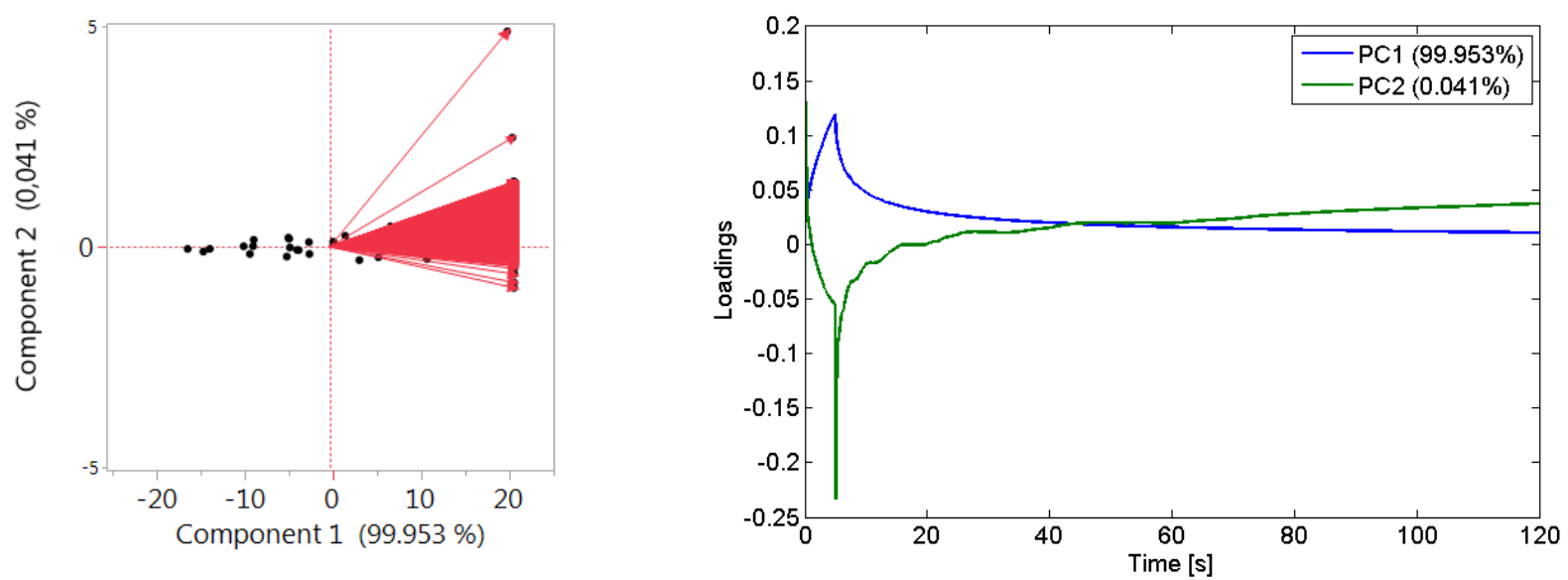

Fig. 5. Biplot of PC1 versus PC2 of the PCA model (left). Black dots and red arrows represent scores and loadings, respectively. Loadings versus time for PC1 and PC2 (right). 


\subsection{1/qirt.2016.026}

\subsubsection{GP model and validation}

For each of the responses, being the scores on PC1 and the scores on PC2, a Gaussian process model was developed. In figure 6, the actual scores on PC1 (left) and PC2 (right) are shown versus the jackknife predicted values of those scores. How well these points lie along the 45 degree diagonal line gives an indication of the goodness-of-fit of the Gaussian process models. An $\mathrm{R}^{2}$-value of 0.9999 and 0.9912 was found for the scores on PC1 and PC2, respectively, indicating the GP models provide a good prediction of the PC1 and PC2 scores. A graphical representation of the GP interpolation models is given in figures 7 and 8 , for PC1 scores and PC2 scores respectively. These surface profiles illustrate how the response changes as a function of the input parameters $k, \rho C_{p}$ and $\varepsilon$.
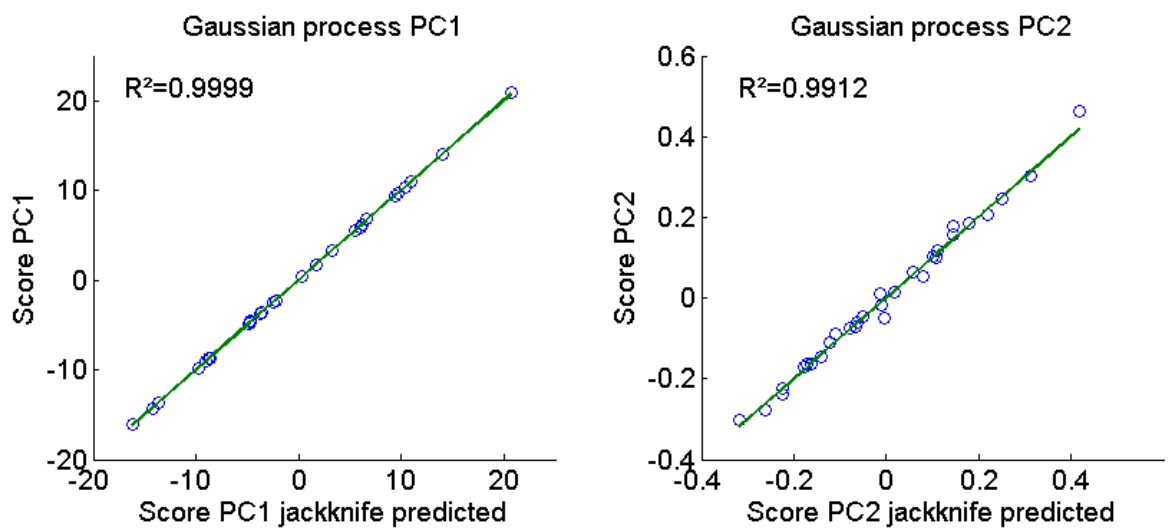

Fig. 6. Actual by predicted PC score plots for the Gaussian process model for PC1 (left) and PC2 (right). The $R^{2}$-value for a $y=x$ fit is given.
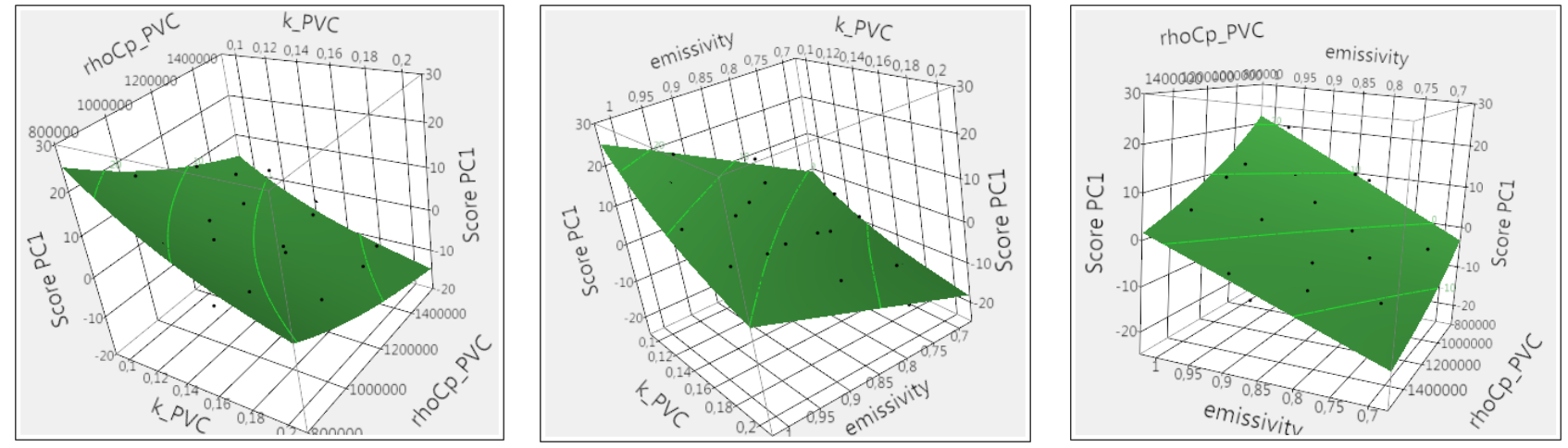

Fig. 7. Graphical representation of the Gaussian process interpolation model for response Score PC1. Black dots indicate the design points. The model is shown for input parameters $k$ and $\rho C_{p}$ (left), $k$ and emissivity (center) and $\rho C_{p}$ and emissivity (right).
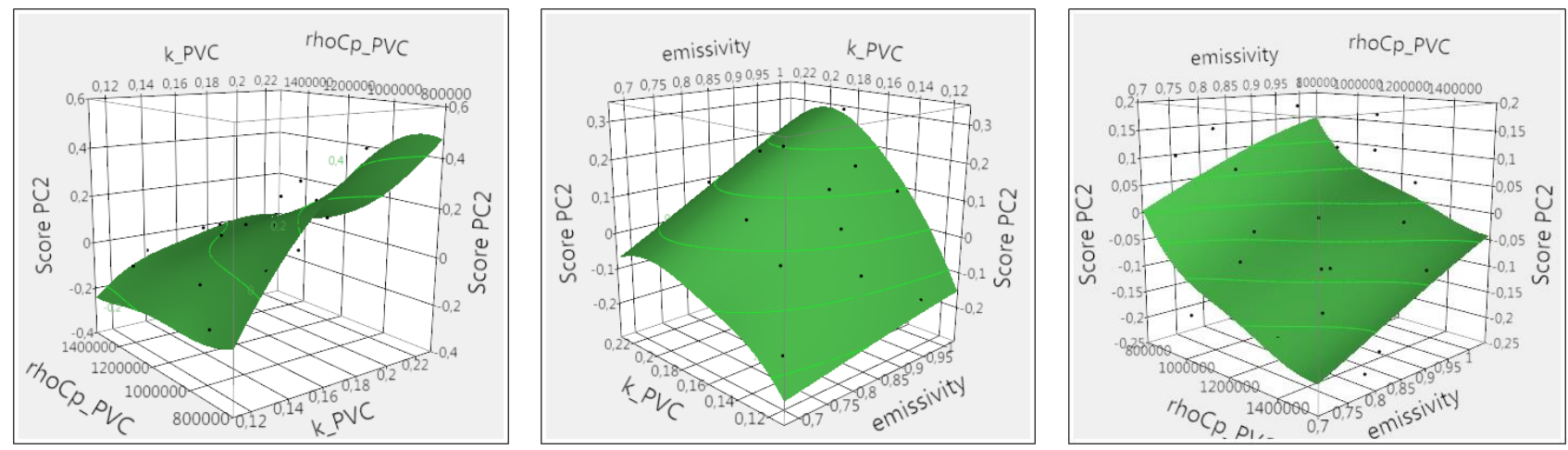

Fig. 8. Graphical representation of the Gaussian process interpolation model for response Score PC2.

Black dots indicate the design points. The model is shown for input parameters $k$ and $\rho C_{p}$ (left), $k$ and emissivity (center) and $\rho C_{p}$ and emissivity (right). 
As mentioned in section 4.4, a space filling design augmentation was performed, adding 10 points to the original Latin hypercube design. These additional design points are indicated in figure 9 (left) in green and red. For all of these points, two temperature-time profiles were obtained: a temperature-time profile obtained from a FEM simulation and a temperature-time profile reconstructed from the PC1 and PC2 scores predicted by the GP models. An example of an overlay of both temperature-time profiles is provided in figure 9 (right) for the setting indicated by the red dot. In table 3 , the values of the input parameters of the 10 validation points are provided, combined with the SSE of the simulated versus reconstructed temperature-time profiles for each point. In order to interpret this SSE value, the SSE values of the simulated profiles compared with the mean simulated profile are also provided. These values indicate that the error introduced in the profiles by reconstructing them from PC1 and PC2 scores obtained from GP model predictions is very limited compared to the overall variation in the temperature-time profiles within the design space.
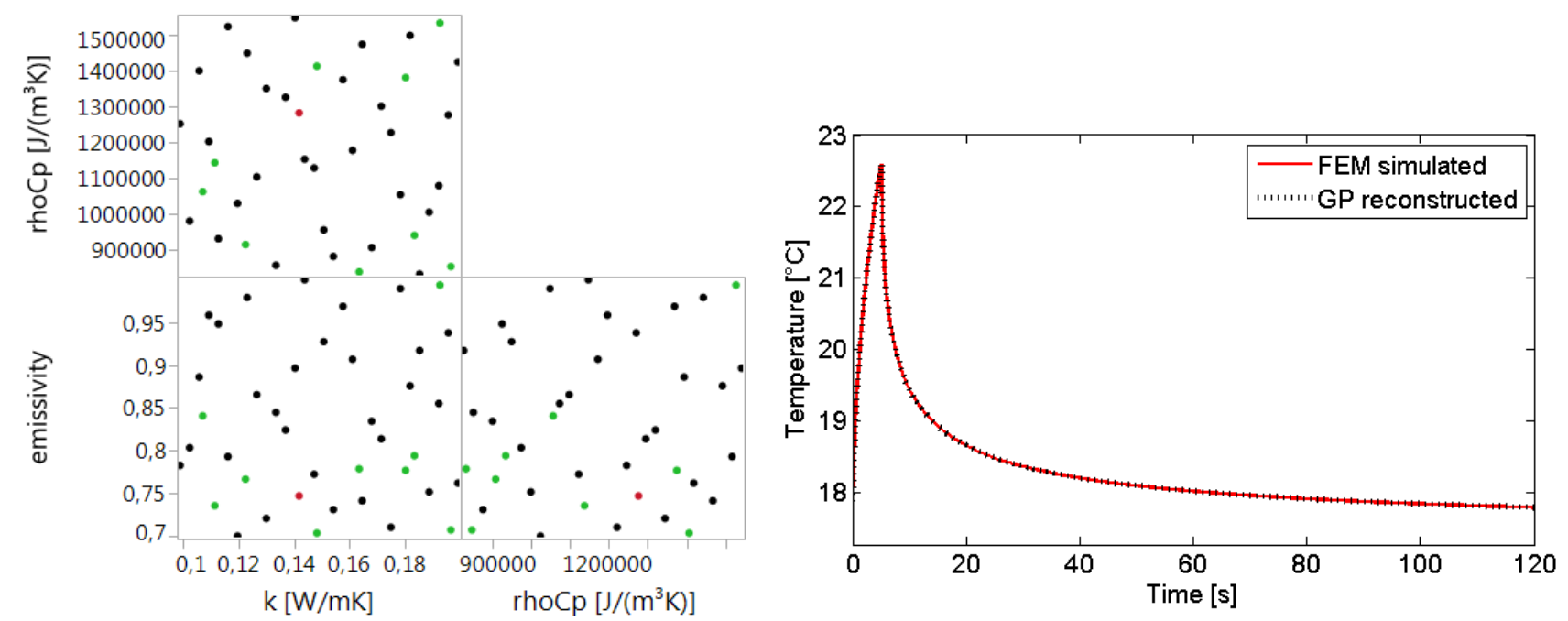

Fig. 9. Left: Space filling design after design augmentation. The green and red dots represent the 10 augmented points. Right: The model validation is illustrated for the combination of material parameters indicated by the red dot.

Table 3. Values of the input parameters at the augmented design points (columns 1-3), SSE of FEM simulated profiles versus profiles reconstructed based on the GP predicted PC1 and PC2 scores (column 4) and SSE of FEM simulated profiles versus mean FEM simulated profile (column 5).

\begin{tabular}{ccccc}
\hline $\begin{array}{c}\text { Thermal } \\
\text { conductivity } \boldsymbol{k} \\
[\boldsymbol{W} / \boldsymbol{m} \cdot \boldsymbol{K})]\end{array}$ & $\begin{array}{c}\text { Volumetric heat } \\
\text { capacity } \\
\boldsymbol{\rho} \boldsymbol{C}_{\boldsymbol{p}}\left[\boldsymbol{J} /\left(\boldsymbol{m}^{\mathbf{3}} \cdot \boldsymbol{K}\right)\right]\end{array}$ & Surface emissivity & $\begin{array}{c}\text { SSE simulated } \\
\text { versus } \\
\text { reconstructed }\end{array}$ & $\begin{array}{c}\text { SSE simulated } \\
\text { versus mean } \\
\text { simulated }\end{array}$ \\
\hline 0.194 & 1534239.112 & 0.994 & & 23.845 \\
0.197 & 855071.271 & 0.707 & 0.006 & 59.093 \\
0.113 & 1144508.517 & 0.736 & 0.026 & 0.115 \\
0.164 & 840202.186 & 0.779 & 0.004 & 2.896 \\
0.184 & 941962.334 & 0.794 & 0.005 & 8.995 \\
0.149 & 1413869.194 & 0.704 & 0.003 & 160.846 \\
0.181 & 1382006.451 & 0.777 & 0.003 & 143.774 \\
0.143 & 1283711.307 & 0.747 & 0.002 & 52.632 \\
0.108 & 1064079.767 & 0.841 & 0.002 & 121.105 \\
0.124 & 916200.262 & 0.767 & 0.006 & 36.402 \\
\hline
\end{tabular}




\subsection{1/qirt.2016.026}

\subsection{Discussion}

In this study, an efficient approach towards heat transfer modeling of active thermography based on the DACE framework was proposed. This approach was developed especially for deterministic computer experiments, avoiding the need for replications in the design. This way, a more efficient sampling of the design space was possible, using a Latin hypercube design. A Gaussian process model was developed to interpolate between the FEM simulations performed at the design points. Since Gaussian process models have been developed for predicting a single output variable and since the responses of heat transfer simulations of active thermography are temperature profiles as a function of time, it was necessary to 'summarize' the profiles in a limited number of parameters. A Principal Component Analysis was used for this purpose and the temperature-time profiles were represented by two principal components. As shown in figure 9 and table 3, the Gaussian process models were able to predict PC1 and PC2 scores that resulted in reconstructed temperature-time profiles in accordance with FEM simulation results. The Gaussian process models can thus be used to obtain temperature-time profiles at any arbitrary combination of input parameters within the limits of the design space, without having the need to run time-consuming FEM simulations. Since the entire profile is obtained as a response of the Gaussian process model, further analysis towards e.g. defect detection and quantification is possible.

To illustrate the approach, thermo-physical properties of the studied PVC sample were used as input parameters in the GP models. Depending on the application, it can be interesting to study other input parameters related to e.g. sample/defect geometry, experimental settings (amplitude, frequency, ...) etc. An inverse approach, namely determining the thermo-physical properties based on a measured temperature-time profile, might also be of interest. However, this requires that there exists a unique solution (=unique combination of thermo-physical properties) resulting in a specific temperature-time profile.

The DACE approach was tested for a FEM model of the heat transfer in a PVC plate with a relatively simple geometry. FEM models of heat transfer are expected to be even more complex, and thus more computationally intensive, when applying them to agro-food products, which are inherently complex and prone to biological variability. That is why the DACE approach is particularly, but not exclusively, of interest for active thermography research in this sector.

\section{Conclusion}

In this study, a finite element model was developed to model the heat transfer processes occurring in a PVC sample during a pulsed thermography inspection. A model including a simulation of the natural convection in the air surrounding the sample was proposed. Although there is an overshoot in the maximum temperature predicted by the model, it describes the cooling profile of sound regions and $1 \mathrm{~mm}, 2 \mathrm{~mm}$ and $3 \mathrm{~mm}$ deep defects well. Since this finite element model was computationally intensive to solve $( \pm 30$ minutes on a $3.40 \mathrm{GHz}$ processor), an efficient way to approach the model was proposed. Finite element simulations were performed at a limited number of well-chosen combinations of thermal conductivity-, volumetric heat capacity- and emissivity values. A Principal Component Analysis was performed on the resulting temperature-time responses of the sound regions and two principal components (PC1 and PC2) were selected to describe the variation in the profiles. Two Gaussian process models were built, interpolating between the responses of the well-chosen simulations and predicting the values of respectively PC1 and PC2 for every arbitrary combination of material properties within the limits of the design space. The Gaussian process models were validated at 10 space filling points that were added to the original design. Low sum of squared error values were obtained between temperature profiles obtained from finite element simulations performed at the validation points and temperature profiles reconstructed based on PC scores predicted by the Gaussian process model at those points. The proposed approach is particularly, yet not exclusively, of interest for active thermography research in the agro-food sector, since agro-food products are inherently complex and prone to biological variability and heat transfer models for these products are expected to be of high complexity.

\section{Acknowledgements}

The authors wish to thank the European Union's Seventh Framework Programme for research, technological development and demonstration PicknPack project under grant agreement no 311987, the Institute for the Promotion of Innovation by Science and Technology in Flanders, Belgium (IWT) Ultraseal project under grant agreement no 140313 and the Industrial Research Fund (IOF) for the financial support. 


\section{REFERENCES}

[1] Maldague X. P., "Theory and practice of infrared technology for nondestructive testing". Wiley, New York, 2001.

[2] Holland, S. D., Reusser, R. S., Material Evaluation by Infrared Thermography, Annual Review of Materials Research - Vol. 46, pp. 5.1-5.17, 2016.

[3] Ibarra-Castanedo, C., Tarpani, J. R., Maldague, X. P. V., Nondestructive testing with thermography, European Journal of Physics - Vol. 34, pp. S91-S109, 2013.

[4] Kylili, A., Fokaides, P. A., Christou, P., et al., Infrared thermography (IRT) applications for building diagnostics: A review, Applied Energy - Vol. 134, pp. 531-549, 2014.

[5] Ibarra-Castanedo, C., Avdelidis, N. P., Grinzato, E. G., Bison, P. G., Marinetti, S., Plescanu, C. C., Bendada, A., Maldague, X. P., Delamination detection and impact damage assessment of GLARE by active thermography, International Journal of Materials and Product Technology - Vol. 41, no 1-4, pp. 5-16 (2011).

[6] Kaczmarek, M., Nowakowski, A., Active IR-Thermal Imaging in Medicine, Journal of Nondestructive Evaluation - Vol. 35, no 1, 2016.

[7] Sfarra, S., Theodorakeas, P., Ibarra-Castanedo, C., Avdelidis, N. P., Paoletti, A., Paoletti, D., Hrissagis, K., Bendada, A., Koul, M., Maldague, X., Evaluation of defects in panel paintings using infrared, optical and ultrasonic techniques, Insight - Non-Destructive Testing and Condition Monitoring - Vol. 54, no. 1, pp. 21-27, 2012.

[8] Lahiri, B. B., Bagavathiappan, S., Reshmi, P. R., Philip, J., Jayakumar, T., Raj, B., Quantification of defects in composites and rubber materials using active thermography, Infrared Physics \& Technology - Vol. 55, pp. 191199, 2012.

[9] Chu, T., Mahajan, A., DiGregorio, A., Russell, S. S., Determination of optimal experimental parameters for transient thermography imaging using finite-element models, The Imaging Science Journal - Vol. 53, no. 1, pp. 20-26, 2005.

[10] Susa, M., Maldague, X., Svaic, S., Boras, I., Bendada, A. "The influence of surface coatings on the differences between numerical and experimental results for samples subject to a pulse thermography examination", Proceedings of $9^{\text {th }}$ Quantitative InfraRed Thermography conference, paper QIRT2008 Krakow (Poland), 2008.

[11] Hernandez-Valle, S., Peters, K., Numerical simulation of phase images and depth reconstruction in pulsed phase thermography, Measurement Science and Technology - Vol. 26, pp. 1-12.

[12] Baranowski, P., Mazurek, W., Witowska-Walczak, B., Slawinski, C., Detection of early apple bruises using pulsed-phase thermography, Postharvest Biology and Technology - Vol. 53, no. 3, pp. 91-100, 2009.

[13] Kim, G., Kim, G.-H., Park, J., Kim, D.-Y., Cho, B.-K., Application of infrared lock-in thermography for the quantitative evaluation of bruises on pears, Infrared Physics \& Technology - Vol. 63., pp. 133-139, 2014.

[14] Senni, L., Ricci, M., Palazzi, A., Burrascano, P., Pennisi, P., Ghirelli, F., On-line automatic detection of foreign bodies in biscuits by infrared thermography and image processing, Journal of Food Engineering - Vol. 128, pp. 146-156, 2014

[15] Sacks, J., Welch, W. J., Mitchell, T. J., Wynn, H. P., Design and Analysis of Computer Experiments, Statistical Science - Vol. 4, no. 4, pp. 409-435, 1989.

[16] Husslage, B. G. M., Rennen, G., van Dam, E. R., den Hertog, D., Space-filling Latin hypercube designs for computer experiments, Optimization and Engineering - Vol. 12, no. 4, pp. 611-630, 2011.

[17] Jones, B., Johnson, R. T., Design and Analysis for the Gaussian Process Model, Quality and Reliability Engineering International - Vol. 25, no. 5, pp. 512-524, 2009.

[18] ASTM C177-13, Standard Test Method for Steady-State Heat Flux Measurements and Thermal Transmission Properties by Means of the Guarded-Hot-Plate Apparatus, ASTM International, West Conshohocken, PA, 2013, www.astm.org.

[19] Thoen, J., Glorieux, C., Leys, J., Differential Adiabatic Scanning Calorimeter, Patent no. WO/2012/103601.

[20] ASTM E1933-14, Standard Practices for Measuring and Compensating for Emissivity Using Infrared Imaging Radiometers, ASTM International, West Conshohocken, PA, 2014, www.astm.org.

[21] Incropera, F. P., DeWitt, D. P., "Fundamentals of heat and mass transfer". John Wiley \& Sons, New York, 2002.

[22] Datta, A. K., "Biological and bioenvironmental heat and mass transfer". Marcel Dekker, New York, 2002.

[23] Acheson, D. J., "Elementary fluid dynamics". Oxford University Press Inc., New York, 1990.

[24] Jolliffe, I. T., "Principal Component Analysis". Springer, New York, 2002.

[25] Naes, T., Isaksson, T., Faern, T., Davies, T., "A user-friendly guide to Multivariate Calibration and Classification". NIR Publications, Chichester, 2002.

[26] Lekivetz, R., Jones, B., Fast flexible space-filling designs for nonrectangular regions, Quality and Reliability Engineering International - Vol. 31, no. 5, pp. 829-837, 2015. 